\title{
PREDIÇÃO DA COLEDOCOLITÍASE PELA ASSOCIAÇÃO DE INDICADORES CLÍNICOS E LABORATORIAIS EM DOIS MOMENTOS DO PRÉ-OPERATÓRIO DA COLECISTECTOMIA
}

\author{
PREDICTION OF PREOPERATIVE CHOLEDOCHOLITHIASIS BY THE \\ ASSOCIATION OF CLINICAL AND LABORATORY INDICATORS IN TWO \\ DISTINCT MOMENTS
}

\author{
Newton Pinto Araújo Neto'; 'José Eduardo Gonçalves,TCBC-SP²; \\ Sansom Henrique Bromberg, ACBC-SP; Betty Guz ${ }^{4}$; Arnaldo Zanoto ${ }^{5}$
}

\begin{abstract}
RESUMO: Objetivo: O propósito deste estudo foi determinar a probabilidade de ocorrência de coledocolitíase através do estudo da associação de indicadores clínicos e laboratoriais desta doença em dois momentos do pré-operatório de colecistectomia. Método: Entre março de 2001 e março de 2002, 48 pacientes consecutivos com colelitíase foram submetidos a colecistectomia e colangiografia intra-operatória (CIO). Os pacientes foram divididos em dois grupos, sendo o grupo A constituído por 13 pacientes com coledocolitíase e o grupo B por 35 pacientes sem esta doença. Os pacientes foram investigados quanto aos indicadores clínicos e laboratoriais da coledocolitíase, analisados em dois períodos, tomando como ponto de corte as 48 horas que precederam a cirurgia. Posteriormente, estes indicadores pré-operatórios foram associados na equação da regressão logística em diferentes combinações. Resultados: Utilizando a equação da regressão logística, constatou-se que a associação de dois indicadores clínicos em ambos os períodos (icterícia e sinal de Murphy) e dois laboratoriais ( nível de corte da gama glutamil transpeptidase e bilirrubina direta 48 horas antes da cirurgia) foi a mais adequada para a predição da coledocolitíase. Os valores obtidos por esta equação mostraram concordância com os grupos A e B, de 95,6\%, e discordância de 4,4\% ( $\mathrm{p}=0,0000007$ e $\mathrm{k}=0,89$ ). Esta equação mostrou sensibilidade de 92,3\%, especificidade de $97,0 \%$, valor preditivo positivo de $92,3 \%$ e valor preditivo negativo de $97 \%$. Estes valores foram próximos aos obtidos pela CIO, que mostrou concordância com os grupos estudados de 95,8\%, e discordância de 4,2\% $(\mathrm{k}=0,90)$. Conclusão: Considerando os resultados obtidos, recomenda-se a associação de indicadores da coledocolitíase na equação da regressão logística para estabelecer a probabilidade de ocorrer coledocolitíase associada à colelitíase. A utilização desta equação pode orientar melhor a conduta diagnóstica e terapêutica nesta doença (Rev. Col. Bras. Cir. 2005; 32(1): 41-46).
\end{abstract}

Descritores: Valor preditivo; Coledocolitíase; Colangiografia; Colecistectomia.

\section{INTRODUÇÃO}

Na era laparoscópica, o avanço tecnológico nos testes radiológicos e endoscópicos onerou em muito a investigação de pacientes suspeitos de coledocolitíase ${ }^{1-3}$. Contudo, ainda permanece indefinida, a conduta mais apropriada nesta doença ${ }^{2-8}$.

A associação de indicadores clínicos e laboratoriais da coledocolitíase para o seu diagnóstico pré-operatório constitui estratégia de baixo custo e disponível em centros médicos com poucos recursos diagnósticos ${ }^{1,2,9,10}$. Embora essa associação tenha aumentado a probabilidade de identificar a coledocolitíase, poucos modelos propostos até o presente momento se aproximaram dos resultados da colangiografia intra-operatória (CIO) 1,2,9,11,12. A maioria dos modelos estudados não considerou o fato dos cálculos na via biliar principal (VBP) formarem arranjo mais dinâmico que estático, nem sempre ocasionando sintomas expressivos ${ }^{3,13,14}$. Em doentes pouco sintomáticos, a reavaliação dos indicadores clínicos e laboratoriais poderia contribuir para o diagnóstico pré-opera- tório da coledocolitíase orientando as investigações complementares por métodos radiológicos $2,3,13$.

O propósito deste estudo foi determinar se a associação de indicadores clínicos e laboratoriais obtidos em dois momentos do pré-operatório de colecistectomia é capaz de aumentar a acurácia diagnóstica de coledocolitíase.

\section{MÉTODO}

Entre março de 2001 e março de 2002, 48 pacientes consecutivos com diagnóstico de colelitíase sintomática foram submetidos à colecistectomia e CIO no Serviço de Gastroenterologia Cirúrgica do Hospital do Servidor Público Estadual - "Francisco Morato de Oliveira" (HSPE-FMO). A idade dos pacientes variou de 40 a 84 anos, com média de $61,15 \pm 11,71$ anos e mediana de 60,5 anos. Quatorze pacientes eram do sexo masculino e 34 do feminino. A coledocolitíase, constatada na exploração cirúrgica da VBP (ECVBP), ocorreu em 13 pacientes (27\%), que formaram o grupo $\mathrm{A}$. O grupo $\mathrm{B}$ foi constituído por 35 enfermos que não apresentaram litíase na

1. Mestre pelo Programa de Pós-Graduação em Gastroenterologia Cirúrgica do IAMSPE

2. Orientador do Programa de Pós-Graduação em Gastroenterologia Cirúrgica do IAMSPE

3. Coordenador do Programa de Pós-Graduação em Gastroenterologia Cirúrgica do IAMSPE

4. Doutora em Medicina pelo IAMSPE

5. Prof. Assistente de Técnica Cirúrgica da FMUSP

Recebido em 15/07/2004

Aceito para publicação em 28/09/2004

Trabalho realizado no Programa de Pós-Graduação em Gastroenterologia Cirúrgica do Instituto de Assistência Médica ao Servidor Público Estadual (IAMSPE) e no Serviço de Gastroenterologia Cirúrgica do Hospital do Servidor Público Estadual "Francisco Morato de Oliveira"- HSPE - FMO. 
VBP. O Comitê de Ética em Pesquisa do HSPE-FMO aprovou o protocolo deste estudo sob o número 022/01, e os doentes, devidamente informados dos procedimentos, concordaram com a realização dos mesmos.

A suspeita clínica de colelitíase foi confirmada em todos os doentes pelo estudo ultra-sonográfico, realizado nos últimos 30 dias que antecederam o ato cirúrgico. Foram excluídos do estudo, os doentes submetidos à colangiopancreatografia endoscópica retrógrada (CPER) prévia à cirurgia e aqueles com afecções das vias biliares e fígado não relacionadas à litíase biliar.

Os exames laboratoriais rotineiros foram acrescidos de outros que, em conjunto com alguns dados clínicos, constituíram os indicadores pré-operatórios da coledocolitíase. A escolha desses indicadores foi baseada em alterações clínicas e laboratoriais observadas na coledocolitíase. Os indicadores clínicos estudados foram: idade, sexo, icterícia, colúria, acolia fecal, dor no hipocôndrio direito (HD), sinal de Murphy e febre. Os indicadores laboratoriais selecionados foram: bilirrubina total (BT), bilirrubina direta (BD), bilirrubina indireta (BI), fosfatase alcalina (FA), gama glutamil transpeptidase (GGT), alanina amino transferase (ALT), aspartato amino transferase (AST) e amilase.

O presente trabalho estabeleceu como ponto de corte as 48 horas que precederam à cirurgia ${ }^{15}$, permitindo a análise dos indicadores clínicos e laboratoriais ao longo do tempo divididos em dois períodos assim definidos:

Período 1 - correspondeu ao intervalo de tempo anterior às 48 horas que precederam a cirurgia, variando de 1 a 212 dias, com média de 18,2 dias e mediana de 2,5 dias.

Período 2 - correspondeu ao intervalo de tempo de 48 horas que precederam o ato cirúrgico.

A análise dos indicadores laboratoriais de vários pacientes no período 1 foi prejudicada devido a problemas técnicos e levou à redução dos examinados. No período 2, essa redução no número de pacientes foi mínima.

Todos os 48 pacientes foram submetidos à colecistectomia e CIO conforme técnica padronizada no Serviço ${ }^{16}$. Este exame radiológico foi considerado normal em 33 pacientes $(68,8 \%)$. A suspeita de coledocolitíase recaiu sobre 15 doentes que apresentaram imagem de subtração de contraste no hépato-colédoco associada ou não à passagem mínima de contraste para o duodeno, em VBP maior que $10 \mathrm{~mm}$. Apenas dois desses 15 pacientes $(13,3 \%)$ não confirmaram a suspeita de coledocolitíase à ECVBP. A CIO apresentou sensibilidade e especificidade elevadas, de $100 \%$ e $94,3 \%$, respectivamente. O seu valor preditivo positivo (VPP) foi de $86,7 \%$, enquanto o valor preditivo negativo (VPN) foi de $100 \%$.

O nível de significância adotado para o cálculo dos dados deste trabalho foi de $5 \%(a=0,05)$. O teste exato de Fisher foi usado para as tabelas de contingência $2 \times 2$. O teste U de Mann-Whitney foi aplicado na análise da diferença entre as médias dos indicadores laboratoriais nos grupos A e B.

$\mathrm{O}$ intervalo compreendido entre o menor e o maior valor do indicador laboratorial para $95 \%$ dos pacientes estudados em cada grupo correspondeu ao intervalo de confiança que variou de $-95 \% \mathrm{a}+95 \%$. Este intervalo foi utilizado na análise dos indicadores laboratoriais mais significantes no período 2. Os níveis de corte (NC) desses indicadores laboratoriais foram obtidos da seguinte forma:

$\mathrm{NC}=$ valor de $(-95 \%)$ do grupo $\mathrm{A}+$ valor de $(+95 \%)$ do grupo B

Neste estudo, a equação da Regressão Logística foi utilizada para estabelecer a probabilidade de ocorrer coledocolitíase pela associação dos indicadores clínicos e laboratoriais obtidos em dois momentos do pré-operatório da colecistectomia. A equação da Regressão Logística é expressa da seguinte maneira:

$$
P=\frac{1}{1+\exp \left[\left(b_{0}+b_{1} x_{1}+b_{2} x_{2}+\ldots+b_{n} x_{n}\right)\right]}
$$

Pé uma variável dependente que representa a probabilidade de ocorrer determinado evento. $\mathrm{O}$ valor de $\mathrm{P}$ na equação da regressão logística varia de 0 a 1 , onde 0 representa a ausência do evento e 1 , a sua presença ${ }^{17}$; $b_{0}$ é uma constante; $\mathrm{b}_{1}, \mathrm{~b}_{2}, \ldots, \mathrm{b}_{\mathrm{n}}$ são coeficientes de regressão logística para as respectivas variáveis independentes $\mathrm{x}_{1}, \mathrm{x}_{2}, \ldots, \mathrm{x}_{\mathrm{n}}{ }^{17}$. Estas variáveis, por sua vez, recebem valores qualitativos e/ou quantitativos.

Em dois doentes, o espécime destinado à feitura da GGT se perdeu. Assim, a equação da regressão logística foi aplicada em 46 doentes, e os seus resultados submetidos ao teste do qui-quadrado $\left(\mathrm{c}^{2}\right)$ e ao coeficiente kappa de Cohen.

\section{RESULTADOS}

A média de idade e o sexo dos enfermos de ambos os grupos estudados foi semelhante $(\mathrm{p}=0,74$ e $\mathrm{p}=0,15)$.

No período 1 , apenas a icterícia se associou à coledocolitíase ( $\mathrm{p}=0,004)$. Neste período, a dor no hipocôndrio direito apresentou elevada sensibilidade, porém especificidade desprezível. A acolia fecal mostrou resultado inverso (Tabela 1).

No período 2, apenas o sinal de Murphy se associou à coledocolitíase $(\mathrm{p}=0,015)$

Em ambos os períodos, os doentes com coledocolitíase apresentaram médias dos indicadores laboratoriais superiores às observadas no grupo B, sendo que a GGT apresentou a diferença mais significante (Tabela 2).

Quando os níveis de corte dos indicadores laboratoriais foram comparados com os grupos estudados (Tabela 3), apenas a GGT se associou à coledocolitíase, apresentando os maiores valores de sensibilidade e especificidade $(\mathrm{p}=0,0003)$.

Após diferentes associações de indicadores pré-operatórios na equação da regressão logística (Tabela 4), constatou-se que, para o diagnóstico da coledocolitíase, a melhor associação foi a de dois indicadores clínicos (icterícia e sinal de Murphy em ambos os períodos) e dois laboratoriais (BD período 2 e nível de corte da GGT).

A equação da Regressão Logística para determinar a probabilidade de ocorrer coledocolitíase passou a ser expressa da seguinte forma: 
Tabela 1- Sensibilidade e Especificidade dos Indicadores Clínicos nos Períodos 1 e 2.

\begin{tabular}{lcccccccc}
\hline & \multicolumn{3}{c}{ Período 1 } & \multicolumn{3}{c}{ Período 2 } \\
\cline { 2 - 8 } $\begin{array}{l}\text { Indicadores } \\
\text { clínicos }\end{array}$ & $\begin{array}{c}\text { Pacientes } \\
\mathbf{N}(\boldsymbol{\%})\end{array}$ & $\begin{array}{c}\text { Sen. } \\
\mathbf{\%}\end{array}$ & $\begin{array}{c}\text { Esp. } \\
\mathbf{\%}\end{array}$ & $\mathbf{p}$ & $\begin{array}{c}\text { Pacientes } \\
\mathbf{N}(\boldsymbol{\%})\end{array}$ & $\begin{array}{c}\text { Sen. } \\
\mathbf{\%}\end{array}$ & $\begin{array}{c}\text { Esp. } \\
\mathbf{\%}\end{array}$ & $\mathbf{p}$ \\
\hline Icterícia & $14(29,2)$ & 61,53 & 82,85 & $<0,05$ & $8(16,7)$ & 30,77 & 88,57 & NS \\
Colúria & $8(16,7)$ & 30,77 & 88,57 & NS & $4(8,3)$ & 7,69 & 91,43 & NS \\
Acolia fecal & $5(10,4)$ & 15,38 & 91,43 & NS & $1(2,1)$ & 0 & 97,14 & NS \\
Dor no HD & $46(95,8)$ & 92,30 & 2,85 & NS & $14(29,2)$ & 38,46 & 74,29 & NS \\
S. Murphy & $9(18,8)$ & 30,77 & 85,71 & NS & $5(10,4)$ & 30,77 & 97,14 & $<0,05$ \\
\hline
\end{tabular}

$N$ - número de pacientes; (\%)- porcentagem; HD- hipocôndrio direito; $S$ - - sinal;

Sen.- sensibilidade; Esp.- especificidade; NS- $p>0,05$. Aplicado o teste exato de Fisher.

$$
\begin{aligned}
\mathrm{P}= & \frac{1}{1+\exp \left\{-\left[-184,76+\left(31,1554 . \mathrm{ict}_{1}\right)+\left(-28,761 . \mathrm{ict}_{2}\right)+\left(-54,668 . \mathrm{Mph}_{1}\right)\right.\right.} \\
& \left.\left.+\left(56,4619 . \mathrm{Mph}_{2}\right)+\left(-0,73963 . \mathrm{BD}_{2}\right)+\left(5,58820 . \mathrm{GGT}_{\mathrm{c}}\right)\right]\right\}
\end{aligned}
$$

Onde $\mathrm{P}$ representou a probabilidade de ocorrer coledocolitíase. A sigla exp significou exponencial e $(-184,76)$ correspondeu a constante $\mathrm{b}_{0}$. Os valores numéricos contidos nos parênteses corresponderam aos coeficientes das respectivas variáveis independentes $\mathrm{b}_{0}, \mathrm{~b}_{1}, \ldots, \mathrm{b}_{\mathrm{n}}$, representadas na equação pelos indicadores pré-operatórios ( Ict $_{1}$ - icterícia no período 1 , Ict 2 - icterícia no período 2, $\mathrm{Mph}_{1}$ - sinal de Murphy no período $1, \mathrm{Mph}_{2}$ - sinal de Murphy no período $2, \mathrm{BD}_{2}-\mathrm{BD}$

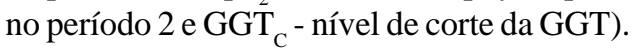

A icterícia, o sinal de Murphy e o nível de corte da GGT $_{C}$ receberam o valor qualitativo de zero para a ausência da coledocolitíase e de um para sua presença. Atribuiu-se valor quantitativo para a BD no período 2 .

$\mathrm{O}$ valor de $\mathrm{P}$ para cada um dos 46 pacientes submetidos à equação da regressão logística mostrou concordância, com os grupos estudados, de $95,6 \%$ e discordância de apenas $4,4 \%(\mathrm{p}=0,0000007)$.

A análise qualitativa da equação da regressão logística tomou como ponto de corte o valor onde $\mathrm{P}=0,5$ (Tabela 5). Esta equação apresentou sensibilidade de $92,3 \%$ e especificidade de $97,0 \%$, enquanto VPP e o VPN foram de $92,3 \%$ e $97,0 \%$, respectivamente $(K=0,89)$. Estes valores foram próximos dos obtidos pela colangiografia intra-operatória que apresentou concordância, com os grupos A e B, de 95,8\% e discordância de 4,2\% (K=0,90).

\section{DISCUSSÃO}

A CIO representou avanço significativo no diagnóstico da coledocolitíase. Antes da sua introdução por Mirizzi em 1932, a hipótese desta afecção era alicerçada somente em parâmetros clínicos. Nestas circunstâncias, a confirmação diagnóstica obtida com a exploração cirúrgica da via biliar principal ocorria em apenas $28 \%$ dos casos $^{6}$. A CIO, entretanto, representa um método invasivo de diagnóstico. Do seu emprego podem resultar adversidades, tais como explorações prolongadas e desnecessárias da via biliar principal e pancreatite aguda, a um tempo aumentando a morbidade per-operatória e retardando a alta hospitalar ${ }^{5}$ ${ }^{9,18}$. No presente estudo, $13,3 \%$ dos casos suspeitos de litíase na VBP pela CIO não foram confirmados à exploração cirúrgica.

Após o advento da cirurgia biliar laparoscópica, intensificou-se a idéia da via mínima de acesso e da agilização técnica do procedimento, objetivando a rápida recuperação pós-operatória do doente. Esta estratégia, contudo, contribuiu para a persistência de algumas controvérsias no tocante a utilização da $\mathrm{CIO}^{19}$.

O aumento no número de CPER pré-operatórias e de colecistectomias associadas a reduções significativas tanto no número de $\mathrm{CIO}$ quanto de explorações cirúrgicas da VBP suscitaram novas tendências, caracterizando sensível mudan-

\begin{tabular}{|c|c|c|c|c|c|c|c|c|}
\hline \multirow[b]{2}{*}{$\begin{array}{l}\text { Indicadores } \\
\text { laboratoriais }\end{array}$} & \multicolumn{3}{|c|}{ Período 1} & \multicolumn{5}{|c|}{ Período 2} \\
\hline & $\begin{array}{l}\text { Média do } \\
\text { Grupo A }\end{array}$ & $\begin{array}{c}\text { Média do } \\
\text { Grupo B }\end{array}$ & $\mathbf{U}$ & $\mathbf{p}$ & $\begin{array}{l}\text { Média do } \\
\text { Grupo A }\end{array}$ & $\begin{array}{c}\text { Média do } \\
\text { Grupo B }\end{array}$ & $\mathbf{U}$ & $\mathbf{p}$ \\
\hline BT & $3,58 \mathrm{mg} / \mathrm{dl}$ & $1,50 \mathrm{mg} / \mathrm{dl}$ & 44,0 & 0,04 & $2,08 \mathrm{mg} / \mathrm{dl}$ & $1,00 \mathrm{mg} / \mathrm{dl}$ & 120,0 & 0,01 \\
\hline BD & $2,39 \mathrm{mg} / \mathrm{dl}$ & $0,97 \mathrm{mg} / \mathrm{dl}$ & 44,0 & 0,04 & $1,56 \mathrm{mg} / \mathrm{dl}$ & $0,58 \mathrm{mg} / \mathrm{dl}$ & 98,0 & 0,003 \\
\hline BI & $1,20 \mathrm{mg} / \mathrm{dl}$ & $0,53 \mathrm{mg} / \mathrm{dl}$ & 45,0 & 0,04 & $0,52 \mathrm{mg} / \mathrm{dl}$ & $0,42 \mathrm{mg} / \mathrm{dl}$ & 168,0 & NS \\
\hline FA & $420,56 \mathrm{U} / \mathrm{L}$ & $230,15 \mathrm{U} / \mathrm{L}$ & 49,0 & NS & $360,92 \mathrm{U} / \mathrm{L}$ & $175,12 \mathrm{U} / \mathrm{L}$ & 190,0 & 0,002 \\
\hline GGT & 477,00 U/L & $177,67 \mathrm{U} / \mathrm{L}$ & 30,0 & 0,008 & 499,92 U/L & 78,73 U/L & 50,0 & 0,00006 \\
\hline ALT & $170,33 \mathrm{U} / \mathrm{L}$ & $66,55 \mathrm{U} / \mathrm{L}$ & 63,0 & 0,02 & $103,58 \mathrm{U} / \mathrm{L}$ & $35,79 \mathrm{U} / \mathrm{L}$ & 76,5 & 0,001 \\
\hline AST & $117,91 \mathrm{U} / \mathrm{L}$ & $75,10 \mathrm{U} / \mathrm{L}$ & 62,5 & 0,03 & 73,17 U/L & 33,25 & 127,5 & NS \\
\hline Amilase & $181,20 \mathrm{U} / \mathrm{L}$ & 79,47 U/L & 74,5 & NS & 68,31 U/L & 63,97 U/L & 195,0 & NS \\
\hline
\end{tabular}
ça na abordagem da coledocolitíase na era laparoscópica ${ }^{19,20}$.

Tabela 2 - Diferenças entre as Médias dos Indicadores Laboratoriais Pré-operatórios nos Grupos A e B nos Períodos 1 e 2.

Grupo A-coledocolitíase; Grupo B-controle; NS- $p>0,05$. Aplicado o Teste U de Mann-Whitney. 
Tabela 3 - Intervalos de Confiança e Nível de Corte dos Indicadores Laboratoriais mais Significantes no Período 2.

\begin{tabular}{|c|c|c|c|c|c|c|c|c|}
\hline \multirow{3}{*}{$\begin{array}{l}\text { Indicadores } \\
\text { laboratoriais } \\
\text { pré-operatórios }\end{array}$} & \multicolumn{4}{|c|}{ Intervalo de confiança } & \multicolumn{4}{|c|}{ Nível de Corte (NC) } \\
\hline & \multicolumn{2}{|c|}{ Grupo A } & \multicolumn{2}{|c|}{ Grupo B } & \multirow[t]{2}{*}{ Valor do NC } & \multirow{2}{*}{$\begin{array}{c}\text { Sen. } \\
\%\end{array}$} & \multirow{2}{*}{$\begin{array}{c}\text { Esp. } \\
\%\end{array}$} & \multirow[t]{2}{*}{$\mathbf{p}$} \\
\hline & $-95 \%$ & $+95 \%$ & $-95 \%$ & $+95 \%$ & & & & \\
\hline FA & $214,85 \mathrm{U} / \mathrm{L}$ & $506,98 \mathrm{U} / \mathrm{L}$ & $140,50 \mathrm{U} / \mathrm{L}$ & $209,73 \mathrm{U} / \mathrm{L}$ & $212 \mathrm{U} / \mathrm{L}$ & 58,33 & 67,65 & NS \\
\hline GGT & $236,39 \mathrm{U} / \mathrm{L}$ & $763,45 \mathrm{U} / \mathrm{L}$ & $53,11 \mathrm{U} / \mathrm{L}$ & $104,34 \mathrm{U} / \mathrm{L}$ & $170 \mathrm{U} / 1$ & $69,23 \%$ & $87,88 \%$ & 0,0003 \\
\hline ALT & $43,36 \mathrm{U} / \mathrm{L}$ & $163,81 \mathrm{U} / \mathrm{L}$ & $25,82 \mathrm{U} / \mathrm{L}$ & $45,75 \mathrm{U} / \mathrm{L}$ & $45 \mathrm{U} / 1$ & $58,33 \%$ & 75,76 & NS \\
\hline
\end{tabular}

-95\%- menor valor do indicador laboratorial para 95\% dos pacientes estudados; +95\%- maior valor do indicador laboratorial para $95 \%$ dos pacientes estudados. Sen.- sensibilidade; Esp.- especificidade.

Aplicado o teste exato de Fisher. NS - p>0,05

Tabela 4 - Análise Estatística da Equação da Regressão Logística Segundo as Variáveis Independentes Utilizadas.

\begin{tabular}{|c|c|c|c|}
\hline $\begin{array}{l}\text { Variáveis independentes utilizadas na } \\
\text { equação da regressão logística }\end{array}$ & $\begin{array}{c}\text { Sensibilidade } \\
\%\end{array}$ & $\begin{array}{c}\text { Especificidade } \\
\%\end{array}$ & $X^{2}$ \\
\hline $\begin{array}{l}\mathrm{Ict}_{1}+\mathrm{ict}_{2}+\mathrm{mph}_{1}+\mathrm{mph}_{2}+\mathrm{BD}_{2}+\mathrm{BI}_{2}+\mathrm{GGT}_{\mathrm{C}} \\
\mathrm{Ict}_{1}+\mathrm{ict}_{2}+\mathrm{mph}_{1}+\mathrm{mph}_{2}+\mathrm{BD}_{2}+\mathrm{GGT}_{\mathrm{C}} \\
\mathrm{Ict}_{1}+\mathrm{ict}_{2}+\mathrm{mph}_{1}+\mathrm{mph}_{2}+\mathrm{GGT}_{\mathrm{C}} \\
\text { mph }_{1}+\mathrm{mph}_{2}+\mathrm{GGT}_{\mathrm{C}} \\
\mathrm{Ict}_{1}+\mathrm{ict}_{2}+\mathrm{GGT}_{\mathrm{C}} \\
\text { Ict }_{1}+\mathrm{ict}_{2}+\mathrm{mph}_{2}+\mathrm{FA}_{\mathrm{C}}\end{array}$ & $\begin{array}{l}92,3 \\
92,3 \\
92,3 \\
76,9 \\
92,3 \\
50,0\end{array}$ & $\begin{array}{l}97,0 \\
97,0 \\
93,9 \\
90,9 \\
84,8 \\
97,1\end{array}$ & $\begin{array}{l}39,05 \\
38,94 \\
36,67 \\
21,90 \\
20,52 \\
17,07\end{array}$ \\
\hline
\end{tabular}

Ict ${ }_{1}$ - icterícia no período 1; ict ${ }_{2}$ - icterícia no período 2; $m_{1} h_{1}$ sinal de Murphy no período 1; mph $_{2}$ - sinal de Murphy no período 2; BD ${ }_{2}$ bilirrubina direta no período 2; $B I_{2}$ - bilirrubina indireta no período 2; $G G T_{c}$ - nível de corte da gama glutamil transferase; FA - nível de corte da fosfatase alcalina; \%- porcentagem; $C^{2}$-valor do qui-quadrado. Aplicado o teste do qui-quadrado.

No futuro, há evidências de que a terapia dos cálculos na via biliar principal se incline para o binômio: redução do emprego da CPER e manutenção da integridade do esfíncter de Oddi ${ }^{8}$. Parece haver pouca justificativa para a indicação de CPER em doentes com baixo risco de apresentar cálculos na via biliar principal ${ }^{19}$. Esta última tendência tem se fortalecido pela crescente utilização da colangio-ressonância como opção à CPER ${ }^{2}$. Entretanto, devido ao alto custo da colangio-ressonância foram desenvolvidos algoritmos para a sua solicitação, baseados no risco individual de ocorrer a coledocolitíase ${ }^{2}$. Portanto, o favorecimento dos procedimentos minimamente invasivos na prática médica vem aumentando o interesse pela associação pré-operatória de indicadores clínicos e laboratoriais com o propósito de estabelecer o risco de litíase na $\mathrm{VBP}^{1-3,5,6,9,11,12,14,21}$.

A incidência da coledocolitíase em doentes com colelitíase submetidos a colecistectomia varia de $4 \%$ a $47 \%$, sendo esta variação dependente da prevalência de litíase vesicular em diferentes regiões ${ }^{6,16,22}$. No HSPEFMO, estudo anterior mostrou incidência de litíase na VBP de $15 \%$, abaixo dos $27 \%$ constatados no presente estudo ${ }^{16}$.

Os cálculos na VBP são mais freqüentes com o avançar da idade, constatando-se associação entre a incidência desta doença e idade superior a 60 anos ${ }^{23,24}$. A média de idade dos doentes com coledocolitíase costuma ser de 9 a 10 anos maior que a dos pacientes sem essa doença ${ }^{21,25}$. No presente estudo, tal fato não foi corroborado, provavelmen- te, devido a elevada faixa etária da população estudada resultando na semelhança entre a média de idade dos pacientes de ambos os grupos - 62 anos no grupo A e 60,8 anos no grupo B.

No grupo A, aproximadamente, $54 \%$ dos pacientes eram do sexo feminino e $46 \%$ do masculino. No grupo B, esta diferença foi ainda maior- $77,14 \%$ de mulheres. Como a litíase vesicular ocorre com frequiência muito maior em mulheres, chamou atenção os percentuais observados no grupo A, embora o cálculo estatístico não tenha evidenciado diferença significante ${ }^{12,21,25,26}$. Este fato já foi constatado, sendo a predisposição do sexo masculino à coledocolitíase atribuída ao maior diâmetro da VBP e à demora em procurar atendimento médico ${ }^{11,16}$.

Tabela 5 - Concordância entre os Resultados da Equação da Regressão Logística Adaptada a Coledocolitíase e os Grupos A e B.

\begin{tabular}{|c|c|c|c|}
\hline $\begin{array}{l}\text { Equação da } \\
\text { regressão logística }\end{array}$ & $\begin{array}{c}\text { Grupo A } \\
\mathbf{N}(\%)\end{array}$ & $\begin{array}{c}\text { Grupo B } \\
\text { N (\%) }\end{array}$ & $\begin{array}{l}\text { Total } \\
\mathbf{N}(\%)\end{array}$ \\
\hline$P>0,5$ & $12(26,1)$ & $1 \quad(2,2)$ & 13 \\
\hline$P<0,5$ & $1 \quad(2,2)$ & $32(69,5)$ & 33 \\
\hline Total & 13 & 33 & $46(100,0)$ \\
\hline
\end{tabular}

$P$ - probabilidade de ocorrer coledocolitíase; $P>0,5$ - coledolitíase; $P \leq 0,5$ - ausência de coledocolitíase; $N$ - número de pacientes; (\%)-percentagem de pacientes.

Aplicado o coeficiente Kappa de Cohen

$K=0,8928$ 
Embora a icterícia seja importante sintoma em pacientes com cálculos na VBP, os pacientes anictéricos também podem apresentar esta doença, levantando dúvidas a respeito do real papel desempenhado por este sintoma no que se refere ao diagnostico precoce da coledocolitíase ${ }^{7,23,25,27,28}$. Nos doentes anictéricos, os exames laboratoriais tornar-seiam ainda mais importantes, pois poderiam identificar anormalidades sugestivas da coledocolitíase 7, 25, 27, 28 .

Estudo da Yale University School of Medicine mostrou que a persistência de anormalidades nos exames laboratoriais pré-operatórios no período de 24 a 48 horas que se seguiram à internação se associaram a maior incidência da coledocolitíase, $31 \%$ contra $8 \%{ }^{15}$. Este estudo, por sua vez, contribuiu para a escolha do ponto de corte adotado no presente estudo, permitindo a análise dos indicadores clínicos e laboratoriais ao longo do tempo divididos em dois períodos. A média de tempo do período 1 foi de 18,2 dias com mediana de 2,5 dias. Os intervalos mais longos observados neste período decorreram do tratamento de pacientes com doenças sistêmicas concomitantes.

Quando os indicadores clínicos estudados foram analisados isoladamente, apenas a icterícia no período $1(\mathrm{p}=$ $0,004)$ e o sinal de Murphy no período $2(\mathrm{p}=0,01)$ se associaram à coledocolitíase. Os valores de sensibilidade e especificidade da icterícia constatados no presente estudo, respectivamente, de $61,54 \%$ e $82,86 \%$, foram semelhantes aos observados por outros autores ${ }^{4,27}$. O sinal de Murphy no período 2 mostrou baixa sensibilidade de $30,77 \%$ porém alta especificidade, de $97,14 \%$.

As médias dos indicadores laboratoriais dos doentes com litíase na VBP foram superiores as do grupo controle em ambos os períodos, entretanto, foi o nível de corte da GGT obtido 48 horas antes da cirurgia, de $170 \mathrm{U} / 1$, que apresentou os maiores valores de sensibilidade e especificidade, respectivamente, de 69,23\% e 87,88\% (p=0,0003). Este fato, vem corroborar com o observado por outros autores, destacando a importância da GGT no diagnóstico da coledocolitíase ${ }^{10,29}$.

A regressão logística vem se destacando dos demais modelos estatísticos como metodologia preditiva para a coledocolitíase $\mathrm{e}^{1,10-12,22}$. Utilizando este modelo, o presente estudo associou quatro indicadores, sendo dois clínicos (icterícia e sinal de Murphy em ambos os períodos) e dois laboratoriais (BD e nível de corte da GGT obtidos 48 horas antes da cirurgia). Embora já se soubesse que tanto a quantidade quanto a combinação de indicadores pré-operatórios interferiam na determinação do risco de apresentar coledocolitíase, ao acrescentar o fator tempo na equação da regressão logística, o presente estudo contribuiu para melhorar a obtenção desse risco apresentando especificidade de $97,0 \%$ e sensibilidade de $92,3 \%$ 15,25, 27,28

Em oposição à maioria dos estudos que associavam os indicadores pré-operatórios atribuindo o mesmo valor à cada um deles, dois estudos ingleses atribuíram valores diferenciados para cada indicador distinguindo a contribuição individual desses indicadores na determinação do risco de apresentar litíase na VBP ${ }^{12,30}$. Embora específicos, a sensibilidade desses dois sistemas de pontuação mostrou ampla variação, de $44 \%$ a $82 \%{ }^{12,30}$. Corroborando com os estudos ingleses, o presente estudo empregou coeficientes de regressão logística para cada um dos indicadores pré-operatórios aprimorando o diagnóstico da coledocolitíase.

No presente estudo ocorreram apenas dois resultados discordantes $(4,4 \%)$, sendo o resultado falso positivo decorrente da compressão do colédoco por intensa reação inflamatória crônica, secundária à presença de cálculo impactado no infundíbulo da vesícula biliar. O resultado falso negativo correspondeu à paciente que apresentava apenas dor no hipocôndrio direito.

Considerando os resultados obtidos, recomenda-se a utilização da equação da regressão logística para a determinação do risco de apresentar coledocolitíase associada à colelitíase. $\mathrm{O}$ uso desta equação possibilitaria a triagem de doentes em algoritmos conforme a probabilidade de ocorrer litíase na VBP. Este dado, nas mãos do cirurgião experiente, orientaria a conduta diagnóstica e terapêutica mais adequada a ser tomada ${ }^{1-3,6,9,11,12,14,21}$. No entanto, é necessário lembrar, que a sua aplicação prática requer o desenvolvimento de um programa computadorizado que simplifique a sua utilização.

\begin{abstract}
Background: The purpose of this study was to determine the probability of choledocholithiasis from the association of clinical and laboratory indicators at two moments of the preoperative phase of cholecystectomy. Methods: Between March 2001 and March 2002, 48 consecutive patients with cholelithiasis were submitted to cholecystectomy and intra-operative cholangiography (IOC). The patients were divided into two groups, with group A composed of 13 patients with choledocholithiasis and group B of 35 patients without this disease. They were investigated by clinical and laboratory indicators of choledocholithiasis, analyzed in two periods, taking as the cut point the 48 hours that preceded the surgery. Later on, these preoperative indicators were associated in the logistic regression equation in different combinations. Results: Using the logistic regression equation, it was found that the association of two clinical indicators in both periods (jaundice and Murphy's sign) and two laboratory indicators (the cut level of gamma glutamyl transpeptidase and direct bilirubin 48 hours before the surgery) was the most suitable for predicting choledocholithiasis. The values obtained by this equation showed an agreement with groups $A$ and $B$ of $95.6 \%$, and a disagreement of $4.4 \%$ ( $p=0.0000007$ and $k=0.89$ ). This equation showed sensitivity of $92.3 \%$, specificity of $97.0 \%$, a positive predictive value of $92.3 \%$ and a negative predictive value of $97 \%$. These values were close to those obtained by the CIO, which showed agreement with the groups studied of $95.8 \%$, and disagreement of $4.2 \%(k=0.90)$. Conclusion: The association of indicators of choledocholithiasis is recommended to establish the probability of there occurring choledocholithiasis associated with cholelithiasis. The use of this equation may provide better guidance for the diagnostic and therapeutic handling of this disease.
\end{abstract}

Key words: Predictive value; Choledocolithiasis; Cholangiography; Cholecystectomy. 


\section{REFERÊNCIAS}

1. Prat F, Meduri B, Ducot B, et al. - Prediction of common bile duct Stones by noninvasive tests. Ann Surg, 1999,229(3):362-368.

2. Demartines N, Eisner L, Schnabel K, et al. - Evaluation of magnetic resonance cholangiography in the management of bile duct stones. Arch Surg, 2000,135(2):148-152.

3. Roston AD, Jacobson IM - Evaluation of the pattern of liver tests and yield of cholangiography in symptomatic choledocholithiasis: a prospective study. Gastrointest Endosc, 1997,45(5):394-399.

4. Bose SM, Mazumdar A, Prakash VS, et al. - Evaluation of the predictors of choledocholithiasis: comparative analysis of clinical, biochemical, radiological, radionuclear, and intraoperative parameters. Surg Today, 2001,31(2):117-122.

5. Sees DW, Martin RR - Comparison of preoperative endoscopic retrograde cholangiopancreatography and laparoscopic cholecystectomy with operative management of gallstones pancreatitis. Am J Surg, 1997,174(6):719-722.

6. Lorimer JW, Lauzon J, Fairfull-Smith RJ, et al. - Management of choledocholithiasis in the time of laparoscopic cholecystectomy. Am J Surg, 1997,174(1):68-71.

7. Leitman IM, Fisher ML, McKinley MJ, et al. - The evaluation and management of known or suspected stones of the common bile duct in the era of minimal access surgery. Surg Gynecol Obstet, 1993,176(6):527-533.

8. Thompson MH - A bile duct stone cannot be found. Br J Surg, 2000,87(5):534-535.

9. Juhasz Csendes A, Burdiles Pinto P, Diaz B JC, et al. - Estudio prospectivo sobre la prevalencia de coledolitiasis de acuerdo a la presencia de factores de riesgo: analisis de 464 colangiografias operatorias. Rev Chil Cir, 1998,50(4):385-393.

10. Trondsen E, Edwin B, Reiertsen O, et al. - Prediction of common bile duct stones prior to cholecystectomy: a prospective validation of a discriminant analysis function. Arch Surg, 1998,133(2):162-166

11. Taylor TV, Armastrong CP, Rimmer S, et al. - Prediction of choledocholithiasis using a pocket microcomputer. Br J Surg, 1988,75(2):138-140.

12. Menezes N, Marson LP, Debeaux AC, et al. - Prospective analysis of a scoring system to predict choledocholithiasis. $\mathrm{Br}$ J Surg, 2000,87(9):1176-1181.

13. Acosta JM, Ledesma CL - Gallstone migration as a cause of acute pancreatitis. N Engl J Med, 1974,290(9):484-487.

14. Neuhaus H, Feussner H, Ungeheuer A, et al. - Prospective evaluation of the use of endoscopic retrograde cholangiography prior to laparoscopic cholecystectomy. Endoscopy, 1992, 24(9):745-749.

15. Cohen ME, Slezak L, Wells CK, et al. - Prediction of bile duct stones and complications in gallstone pancreatitis using early laboratory trends. Am J Gastroenterol, 2001,96(12):3305-3311.

16. Bromberg SH, Waisberg J, Gonçalves JE, et al. - Tratamento cirúrgico da litíase biliar em idosos: experiência em hospital de ensino. Rev Col Bras Cir, 1998,25(3):161-166.
17. Armitage P, Berry G - Statistical methods in medical research. $3^{\text {rd }}$ Edition. Cambridge - Blackwell Science, 1994.

18. Shively EH, Wieman TJ, Adams AL, et al. - Operative cholangiography. Am J Surg, 1990,159(4):380-385.

19. Perissat J, Huibregtse K, Keane FB, et al. - Management of bile duct stones in the era of laparoscopic cholecystectomy. Br J Surg, 1994,81(6):799-810.

20. Cervantes J, Rojas G, Anton J - Conductas actuales ante la litiasis coledociana. Cir Cir, 1999, 67(2):49-53.

21. Grace PA, Qureshi A, Burke P, et al. - Selective cholangiography in laparoscopic cholecystectomy. Br J Surg, 1993,80(7):244246.

22. Barkun AN, Barkun JS, Fried GM, et al. - Useful predictors of bile duct stones in patients undergoing laparoscopic cholecystectomy. McGill Gallstone Treatment Group. Ann Surg, 1994,220(1):32-39.

23. Wilson TG, Hall JC, Watts JM - Is operative cholangiography always necessary? Br J Surg, 1986,73(8):637-640.

24. Hauer-Jensen M, Karesen R, Nygaard K, et al. - Prospective randomized study of routine intraoperative cholangiography during open cholecystectomy: long-term follow-up and multivariate analysis of predictors of choledocholithiasis. Surgery, 1993,113(3): 318-323.

25. Lacaine F, Corlette MB, Bismuth H - Preoperative evaluation of the risk of common bile duct stones. Arch Surg, 1980,115(9):1114-1116.

26. Pernthaler H, Sandbichler P, Schmid T, et al. - Operative cholangiography in elective cholecystectomy. Br J Surg, 1990,77(4):399-400.

27. Samaniego Ayala C - Probabilidades de litiasis de la via biliar principal en pacientes con colecistitis calculosa. Rev Argent Cir, 1999,76(5):125-129.

28. Del Santo P, Kazarian KK, Rogers F, et al. - Prediction of operative cholangiography in patients undergoing elective cholecystectomy with routine liver function chemistries. Surgery, 1985,98(1):7-11.

29. Pereira- Lima JC, Jakobs R, Busnello JV, et al. - The role of serum liver enzymes in the diagnosis of choledocholithiasis. Hepatogastroenterology, 2000, 47(36):1522-1525.

30. Neoptolemos JP, London N, Bailey I, et al. - The role of clinical and biochemical criteria and endoscopic retrograde cholangiopancreatography in the urgent diagnosis of common bile duct stones in acute pancreatitis. Surgery, 1986,100(4):732-742.

Endereço para correspondência:

Newton Pinto Araújo Neto

Rua Paulo Sergio Oliveira Aquino 46, ap- 33 A,

Vila Progresso

07095170 -Guarulhos- SP

e-mail: newtonpneto@directnet.com.br 